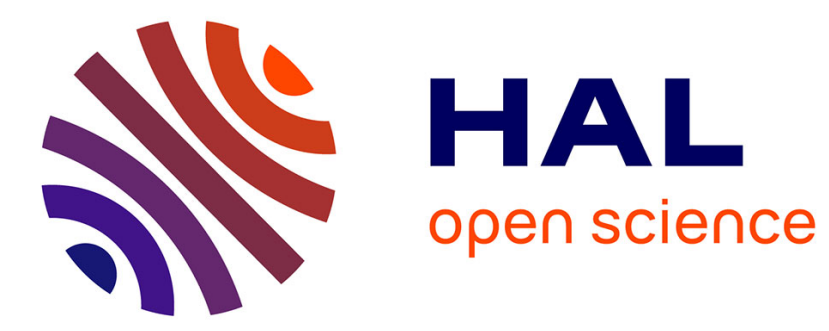

\title{
La régulation des effets de la pornographie
}

Florian Voros

\section{To cite this version:}

Florian Voros. La régulation des effets de la pornographie. Hermès, La Revue - Cognition, communication, politique, 2014, Sexualités, 69. halshs-01276995

\section{HAL Id: halshs-01276995 \\ https://shs.hal.science/halshs-01276995}

Submitted on 6 Mar 2017

HAL is a multi-disciplinary open access archive for the deposit and dissemination of scientific research documents, whether they are published or not. The documents may come from teaching and research institutions in France or abroad, or from public or private research centers.
L'archive ouverte pluridisciplinaire HAL, est destinée au dépôt et à la diffusion de documents scientifiques de niveau recherche, publiés ou non, émanant des établissements d'enseignement et de recherche français ou étrangers, des laboratoires publics ou privés. 


\section{Florian Voros, « La régulation des effets de la pornographie »,}

Hermès, $\mathrm{n}^{\circ}$ 69, 2014, p. 125-129.

(dossier « Sexualités » dir. Ernest-Armand Amato, Fred Pailler et Valérie Schafer)

Résumé. De l'invention de l'obscénité comme catégorie de censure (début XIXe) aux premières croisades morales contre la démocratisation de la pornographie (fin XIXe), de nouvelles relations de savoir et de pouvoir se tissent autour de l'enjeu des effets de la pornographie sur les corps et les comportements des publics prétendus fragiles. Retracer l'émergence de ce dispositif de régulation des images et des corps permet en retour d'éclairer ses reconfigurations contemporaines: comment se réactualisent ces relations de savoirpouvoir à l'occasion des paniques morales concernant les «tournantes » ou l'«addiction sexuelle $» ?$

Mots-clés : censure, publics, savoirs, effets, affects.

Bio. Florian Voros est doctorant en sociologie à l'Institut de recherche interdisciplinaire sur les enjeux sociaux (Iris), École des hautes études en sciences sociale (Ehess), Paris. Sa recherche doctorale porte sur les usages sociaux de la pornographie audiovisuelle et les constructions de la masculinité. Il est également attaché temporaire d'enseignement de recherche en sciences de l'information et de la communication à l'Université Paris 8 Vincennes - Saint-Denis, et membre des comités de rédaction des revues Porn Studies et Poli - Politique de l'image. 
La sexualité est une dimension centrale des théories scientifiques de la communication qui émergent au XIXe siècle. Williams (1999) a insisté sur l'imbrication du savoir scientifique, $\mathrm{du}$ plaisir sexuel et des rapports de genre dans l'invention des théories et techniques modernes relatives à la compréhension et à l'enregistrement du mouvement humain. Le zoopraxiscope de Muybridge, le kinétoscope d'Edison et la photographie de l'hystérie de Charcot sont, selon cette théoricienne féministe du cinéma, traversés par une même volonté de savoir masculine sur les "vérités » sexuelles confessées par les corps féminins. Revenir sur le moment historique d'invention de la pornographie moderne consiste alors moins pour Williams à écrire un chapitre particulier de l'histoire du cinéma, qu'à reconsidérer la place de la sexualité dans l'invention des théories et techniques cinématographiques modernes.

Cette démarche de recentrement de la sexualité peut être poursuivie, en la transposant du domaine des savoirs relatifs au fonctionnement du «moteur humain» vers celui de la mécanique des «foules» (Mattelart, 1994 : 255-336). Les savoirs sont ici entendus au sens foucaldien comme l'ensemble des pratiques discursives qui visent à connaître les effets de la pornographie sur les corps et les comportements de ses publics. L'invention de l'obscénité comme catégorie de censure au début du XIXe siècle (Kendrick, 1987 ; Hunt, 1993), suivie des premières croisades morales contre la démocratisation de la pornographie à la fin du XIXe siècle (Stora-Lamarre, 1989 ; Sigel, 2000), sont des moments clés dans la formation historique des savoirs sur les effets anomiques des médias dits de masse. La régulation de la pornographie permet d'interroger les dimensions sexuelles des théories scientifiques et des paniques morales qui se focalisent, à partir de la fin du XIXe siècle, sur les « effets directs » des médias (Maigret, 2007 : 45-54).

\section{L'exclusion des publics subalternes.}

Dans le discours des bibliothécaires, des historiens de l'art, des conservateurs de musée, des juristes, des savants et des moralistes qui s'attachent à partir du début du XIXe siècle à penser, classer et réguler les textes, images et objets qui «offensent un sens basique et partagé de l'ordre social» (Peignot, 1806, cité in Hunt, 1993: 14), les termes de pornographie et d'obscénité sont interchangeables. Pour comprendre le sens moderne de ces termes, Linda Williams propose de croiser deux étymologies. L'obscénité est à la fois ce qui est «grossier, vulgaire, qui a trait aux excréments» (obscenus) et ce qui est ou doit «être 
placé hors $(o b)$ de la scène (scena) de la représentation publique » (Williams, 2004 : 174). La combinaison de ces deux acceptions indique comment cette nouvelle catégorie de censure regroupe des objets culturels qui, au-delà de leur grande hétérogénéité ${ }^{1}$, ont en commun de «blesser ouvertement la pudeur», selon la définition de l'obscénité qui s'impose alors dans le dictionnaire Littré.

Dans l'introduction de son ouvrage consacré aux objets "pornographiques » exhumés des ruines de Pompéi, Louis Barré précise que les hommes sans éducation, les femmes et les enfants ne doivent en aucun cas accéder à son contenu, afin que soient préservées « les lois de la décence et de la modestie » (1867, cité in Kendrick, $1987: 15)$. Cette préoccupation pour les effets de la pornographie sur les corps des publics subalternes est associée à la mise en place de stratégies d'exclusion, à travers l'interdiction d'accès aux collections pornographiques des musées et des bibliothèques, des prix de vente élevés, l'usage d'un langage érudit et, surtout, l'instauration d'un contrôle policier sur «l'exposition ou la distribution de pamphlets, chansons, figures ou images contraires aux bonnes mœurs » (Code Pénal de 1810). Dans les colonies britanniques, dans un contexte de démocratisation de la consommation sexuelle à travers la carte postale érotique à partir des années 1880, l'enjeu est plus spécifiquement de prévenir l'accès des hommes non-blancs à des représentations pornographiques de femmes blanches (Sigel, $2000: 877$ ).

Retracer l'émergence de cette catégorie de censure revient à esquisser la formation d'un nouveau «champ de bataille culturel» (Kendrick, 1987: 31), c'est-à-dire d'un combat élitiste qui vise à limiter la visibilité et l'accessibilité des représentations médiatiques de la sexualité. La « nécessité de protéger la société de l'immoralité » (Peignot, 1806, cité in Hunt, 1993 : 16) apparaît alors comme « un projet normatif qui se fonde sur la séparation des publics » (Stora-Lamarre, $1989: 12$ ), autrement dit, sur une « ségrégation politique du regard basée sur [1'âge,] le genre, la classe et la race»(Preciado, 2010 : 27). L'obscénité est un principe d'exclusion des publics subalternes.

1 Au-delà des seules représentations explicites de la sexualité, la pornographie regroupe, lors sa naissance comme catégorie de censure, l'ensemble des textes qui contreviennent aux normes morales de l'espace public bourgeois. 


\section{L'objectivation des effets.}

En même temps qu'elle s'efforce de les maintenir hors de portée des mains et des yeux des publics subalternes, la lutte contre les effets de la pornographie invente un nouveau regard sur les représentations médiatiques de la sexualité. La deuxième condition posée par Louis Barré à la création de catalogues sur les objets «pornographiques » de Pompéi est que « l'homme de science ne sourie ni ne rougisse »(Kendrick, 1987: 15). À la servitude corporelle des publics animalisés ou infantilisés, s'oppose ainsi la maîtrise de soi de l'homme de science, « adulte » et « civilisé ». La distinction entre publics capables d'autocontrôle et publics placés sous tutelle est également au fondement de la réflexion de l'État français sur la censure : « selon les tenants du ministère public, seul le chercheur, l'érudit, l'expert ou le bibliothécaire peuvent résister au péril du livre pornographique. Loin des «masses » et des «foules », le livre appartient à ceux qui ont un jugement et un goût sûrs » (Stora-Lamarre, 1989 : 9). La régulation des images est également une régulation des corps. Et le gouvernement des effets de la pornographie sur les « masses » implique en retour le gouvernement de soi de l'homme de science.

L'objectivation des effets de la pornographie sur les publics subalternes (femmes, jeunesse, classes populaire, populations colonisées) passe par la constitution d'un nouveau sujet de connaissance qui, à la manière du « témoin modeste » critiqué par l'épistémologie féministe des sciences (Haraway: 319), prétend s'abstraire de sa propre corporéité. Le privilège épistémologique accordé à l'homme de science est la connaissance objective et rationnelle des effets charnels de la pornographie sur les «autres ». La «modestie » et la « décence» sont présentées par Louis Barré (Kendrick, 1987 : 15) comme des postures permettant une bonne distance scientifique avec les mauvais effets qu'auraient les mauvaises images lorsqu'elles seraient vues et manipulées par les mauvais publics. En plus d'un nouveau rapport d'exclusion, la lutte contre les effets de la pornographie produit ainsi un nouveau rapport d'objectivation, reposant sur l'articulation des binarismes eux/nous, corps/esprit, émotion/raison et subjectivité/objectivité. 


\section{Un dispositif de régulation biopolitique.}

Ce maillage de relations de savoir-pouvoir forme un dispositif de régulation biopolitique, « comportant des discours, des institutions, des aménagements architecturaux, des décisions règlementaires, des lois, des mesures administratives, des énoncés scientifiques, des propositions philosophiques, morales, philanthropiques, bref : du dit aussi bien que du non dit» (Foucault, 1994[1977]: 299). Il faut insister sur la dimension biopolitique de ce dispositif, au sein duquel la régulation de la visibilité et de l'accessibilité des images pornographiques se justifie par un objectif d' « amélioration » du corps national.

Dans un contexte politique où l'éducation est le « mythe fondateur de la IIIe République », la répression de l'obscène se veut avant tout «éducative»(Stora-Lamarre, 1989: 211). La restriction de l'accès à la pornographie doit permettre à la jeunesse, le futur de la nation, « de se développer normalement, raisonnablement, sainement », comme l'affirme Louis Compte lors du Premier Congrès National contre la pornographie à Bordeaux en 1905. Au récit de l'anomie dans lequel on inscrit alors l'accessibilité croissante des cartes postales érotiques, s'oppose un récit du progrès et de la civilisation à travers la démocratisation d'une culture écrite, froide et rationnelle.

Dans un contexte où l'" on définit pour la première fois une morbidité propre au sexuel », où « le sexe apparait comme un champ de haute fragilité pathologique », comme une «surface de répercussion pour d'autres maladies» mais aussi comme un «foyer de nosographie propre, celle de l'instinct, des penchants, des images, de la conduite » (Foucault, $1976: 90$ ), la lutte contre les effets de la pornographie est indissociablement un projet éducatif et sanitaire. Les paradigmes de la «dégénérescence» et de la «perversion» fournissent, notamment sous la plume d'Émile Pourésy, le cadre théorique des premières théories médicales des effets de la pornographie, dont la visibilité croissante participerait d'une usure des sens et d'une fatigue des organismes, conduisant à des pathologies héréditaires. Le déploiement d'un réseau de relations de savoir-pouvoir autour des effets de la pornographie est orienté vers la surveillance des sensations corporelles potentiellement pathologiques (donc réformables et soignables) ainsi que vers la protection et la promotion de la vitalité du corps social, de la « nation » et de la « race ». 


\section{De l'obscénité à la surscénité.}

Selon Foucault, un dispositif advient pour « remplir une fonction stratégique », souvent pour « répondre à une urgence » (1994[1977] : 299). Cette urgence est posée à la fin du XIXe siècle par la visibilité et l'accessibilité croissantes de la pornographie, dans un contexte de rigidification des normes de pudeur, ainsi que de stabilisation d'un ordre social reposant sur des hiérarchies d'âge, de genre, de race et de classe. Foucault poursuit en expliquant que les dispositifs survivent ensuite, en se reconfigurant, aux intentions qui ont présidé à leur mise en place.

Williams (2004) avance que les sociétés européennes et nord-américaines ont, dans les années 1970, changé de régime de visibilité et d'accessibilité médiatique de la sexualité, en passant de l'obscénité à la surscénité ${ }^{2}$. « La surscénité n'est pas la fin de l'obscénité » (ibid. : 165) mais plutôt une crise définitionnelle de l'obscénité. Les critères de censure font désormais l'objet de controverses publiques à répétition, dans un contexte de "politisation croissante des représentations médiatiques de la sexualité » et de «prolifération du discours sexuel » (ibid. : 165). Quelles sont dès lors les reconfigurations contemporaines des relations de savoir-pouvoir dont nous venons de faire la généalogie?

Le lien entre les intensités affectives de la pornographie, la santé individuelle et la santé du corps social reste au XXIe siècle au centre du problème. Si le langage médical - par exemple celui de l'addiction et de la compulsion sexuelles (Voros, 2009) qui découleraient d'un engagement excessif dans les mondes de la pornographie en ligne - est largement contesté, l'enjeu du débat reste bien la définition publique de la bonne santé et de la rationalité sexuelles. Surtout, l'injonction à l'autocontrôle des publics est un des principaux ressorts des discours de prévention et de guérison de ces « troubles du contrôle sexuel ».

Un autre prolongement historique apparaît dans la primauté de plus en plus marquée du registre positiviste des «preuves scientifiques » sur le registre moraliste des «valeurs » dans la dénonciation des dangers de la pornographie (Williams, 2004). Cette technicisation des modes de justification de la censure accompagne un effort scientifique de quantification de plus en plus précise de l'effet immédiat de la pornographie, de l'administration du 
questionnaire dans la psychologie béhavioriste à la mesure de l'afflux sanguin dans les organes génitaux avec le pléthysmographe en psychiatrie.

La dénonciation des effets de la pornographie se reformule à partir des années 1970 en une dénonciation des violences masculines envers les femmes que ces images encourageraient (Bronstein, 2011). Dans un contexte d'accessibilité croissante des jeunes à la pornographie avec le développement d'Internet, cette thématique est reprise dans les années 2000 par une série de rapports officiels qui, faute de preuves, en appellent à un «principe de précaution » (Kriegel, $2002: 66$ ). Les connotations raciales et de classe de cette préoccupation éducative et sanitaire de l'État se trouvent explicitées au même moment dans la médiatisation des « tournantes » (Saadaoui, 2010), à travers laquelle les traits du «jeune de banlieue » en viennent à dessiner les nouveaux contours du problème.

Le dispositif de régulation biopolitique des effets de la pornographie a survécu en s'adaptant aux transformations politiques et culturelles. Les relations de savoir-pouvoir agencées par ce dispositif se sont recomposées : les théories des «effets directs » et les contours du public « problématique » ont évolué, tandis que la pornographie reste pensée comme un enjeu de gouvernement de soi et de gouvernement des autres.

\section{$\underline{\text { Références citées }}$}

BRONSTEIN C., Battling Pornography. The American Feminist Anti-Pornography Movement, 1976-1986, Cambridge, Cambridge University Press, 2011.

Foucault, M., Histoire de la sexualité, tome I: La volonté de savoir, Paris, Gallimard, 1976.

FoucAult, M. «Le jeu de Michel Foucault», in Dits et écrits, tome II, Paris, Gallimard, 1994 [1977], p. 298-329.

HARAWAY, D., Manifeste cyborg et autres essais: sciences, fictions, féminismes. Paris, Exils, 2007.

Hunt, L., The Invention of Pornography: Obscenity and the Origins of Modernity, 15001800. New York, Zone Books, 1993.

KENDRICK, W., The Secret Museum : Pornography in Modern Culture. New York, Viking, 1987.

KRIEGEL B., La violence à la télévision : rapport à M. Jean-Jacques Aillagon ministre de la culture et de la communication, Paris, Ministère de la culture et de la communication, 2002. 
MAIGRET É., Sociologie de la communication et des médias, $2^{\mathrm{e}}$ éd., Paris, Armand Colin, 2007.

Mattelart, A. L'invention de la communication, $3^{\mathrm{e}}$ éd., Paris, La Découverte, 2011.

PRECIADO, B. « The Architecture of Porn. Museum Walls, Urban Detritus and Stag Rooms for porn-prosthetic Eyes ", in STÜTTGEN T., Post Porn Politics. Queer-Feminist Perspective on the Politics of Porn Performance and Sex-Work as Culture Production, Berlin, b_books, 2010, p. 23-33.

SAADAOUI L., Traitement et interventions médiatiques autour des "tournantes " en France, Thèse de doctorat, Université Paul Verlaine de Metz, 2010.

SigeL, L., "Filth in the Wrong People's Hands : Postcards and the Expansion of Pornography in Britain and the Atlantic World, 1880-1914 », Journal of Social History, vol. $33, n^{\circ} 4,2000$, p. 859-885.

StORA-LAMARRE, A., L'Enfer de la IIIe République : censeurs et pornographes (1881-1914), Paris, Imago, 1989.

VOROS, F., «L'invention de l'addiction à la pornographie », in Sexologies, vol. 18, n 4, 2009, p. 270-276.

Williams, L., Hard Core. Power, Pleasure and the "Frenzy of the Visible ". $2^{\mathrm{e}}$ éd. Berkeley, University of California Press, 1999.

Williams, L., "Second Thoughts on Hard Core : American Obscenity Law and the Scapegoating of Deviance », in CHURCH-GIBSON P., More Dirty Looks : Gender, Pornography and Power, Londres, British Film Institute, 2004, p. 165-175. 